HEROES AND MARTYRS OF QUALITY AND SAFETY

\title{
Too good to last: did Cleveland Health Quality Choice leave a legacy and lessons to be learned?
}

\author{
D Neuhauser, D L Harper
}

Qual Saf Health Care 2002;1 1:202-203

T his is the story of the martyrdom of an idea, an organization that embodied that idea, its director, and a group of staff and volunteers committed to making it work. The idea is that the comparable outcomes of hospital care should be measured and publicly reported so people could choose high quality care. This idea was embodied in a small organization called Greater Cleveland Health Quality Choice (GCHQC) and its director Dwain Harper

\section{GREATER CLEVELAND HEALTH QUALITY CHOICE WAS AN IDEA TOO GOOD TO LAST!}

In the 1980s large and small businesses in Cleveland, Ohio paying for health insurance for their employees and families were experiencing annual double digit inflation for their costs for employee health care in the 30 or more hospitals in the area. ${ }^{*}$ Like sensible businessmen and women, they decided to select those hospitals that had low costs and could demonstrate high quality. They implemented strategies to encourage their employees to use them. In spite of claims to the contrary, Cleveland employers did not want to purchase just the least expensive hospital care; they wanted to purchase value, a functional balance between low cost and high quality. Quality should matter and employers would measure it and make the results publicly available.

\section{THE PUBLIC SHOULD KNOW}

Collectively, in the 1980s, employers had enough power in this marketplace (Cleveland) to demand that hospitals submit data amenable to reporting standardized quality measures which would be made public so people could make informed decisions and choose the hospital having the best value. Physicians and hospitals found it wise to participate in this initiative and assumed leadership roles in the program's creation and evolution. Businesses saw this as a desirable advantage and partnered with providers in this unique program.

By 1990, standardized data were being collected independently which enabled reporting of severity adjusted mortality rates, cesarean section deliveries, vaginal birth after prior cesarean section delivery, patient satisfaction, and length of stay. Every 6 months this information was shared with employers in comprehensive reports and with consumers in public report cards. In these reports hospitals were named and performance statistics were provided, and some of the information routinely made the front page of the local newspapers and mention on local TV. Table 1 illustrates mortality performance for critical care patients for a 1 year period

\footnotetext{
*For those not familiar with the economics of health care in the USA it is important to know that, for working age employees and their families, health insurance is paid through their employer. Because the companies pay, they see a clear need to control these costs and keep their employees content. The hospitals are independent corporations and free to negotiate over the price of care with each payer. The relative bargaining power between these two parties varies.
}

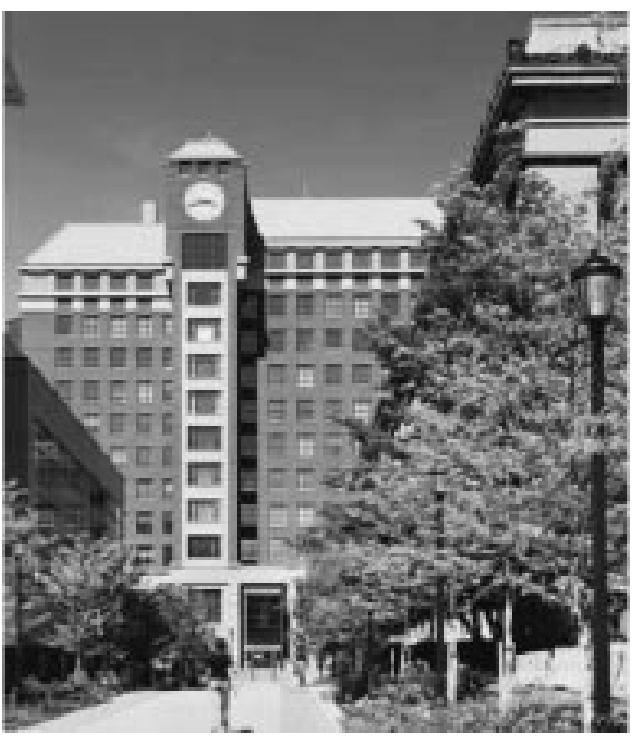

broken down into 6 month intervals. The information is displayed in a format adopted by the Cleveland Plain Dealer for public reporting.

A lot of creativity, effort, and expense went into the severity adjustment methods, public reporting format, data display, and analysis. The objectives of these efforts were to satisfy conventional criteria of the biostatisticians, physicians, and clinicians and be understandable to the public.

One of the legacies of GCHQC is the rich and robust database of information derived from this effort. Millions of clinical data elements about tens of thousands of hospital admissions were collected. This resource, made available to researchers under strict protocol, has spawned over 30 articles appearing in the refereed scientific literature and more are in progress (see QSHC website (http:// www.qualityhealthcare.com) for partial inventory).

Table 1 Public report appearing in newspaper showing mortality rate for critical care patients (two 6 month periods)

\begin{tabular}{lll}
\hline Hospital & Report 11 & Report 12 \\
\hline Allen Memorial & $\Leftrightarrow$ & $\Leftrightarrow$ \\
Cleveland Clinic & $\Uparrow$ & $\Uparrow$ \\
Community Health Ptnr & $\Leftrightarrow$ & $\Leftrightarrow$ \\
EMH Regional & $\Leftrightarrow$ & $\Downarrow$ \\
Euclid & $\Leftrightarrow$ & $\Downarrow$ \\
Fairview & $\Leftrightarrow$ & $\Leftrightarrow$ \\
Hillcrest & $\Leftrightarrow$ & $\Leftrightarrow$ \\
Huron & $\Leftrightarrow$ & $\Leftrightarrow$ \\
\hline
\end{tabular}

$\Uparrow=$ performance better than expected; $\Leftrightarrow=$ performance as expected; $\Downarrow=$ performance worse than expected. 
Table 2 Aggregate hospital performance improvement 1993-1997

\begin{tabular}{lll}
\hline Diagnosis/procedure & $\begin{array}{l}\text { Mortality } \\
\text { rates }\end{array}$ & $\begin{array}{l}\text { Length of } \\
\text { stay }\end{array}$ \\
\hline Acute myocardial infarction (AMI) & $11.1 \%$ & $21.6 \%$ \\
Coronary artery bypass graft (CABG) & $24.6 \%$ & $12.7 \%$ \\
Congestive heart failure & $34.7 \%$ & $23.4 \%$ \\
Gastrointestinal bleeding & $17.4 \%$ & $21.0 \%$ \\
Lower lowel resectiion & $25.6 \%$ & $20.3 \%$ \\
Chronic obstructive pulmonary disease & $32.3 \%$ & $22.7 \%$ \\
Pneumonia & $23.5 \%$ & $19.4 \%$ \\
Stroke & $10.2 \%$ & $33.7 \%$ \\
\hline
\end{tabular}

However, one of the unfulfilled visions of the architects of the program was the use of the quality reports by citizens to make more informed purchasing decisions. If you had stood on a street corner in Cleveland and asked average pedestrians if they were aware of these reports and used the report to make a healthcare decision, perhaps one in 100 would have said "yes". On the contrary, every hospital administrator and senior physician in town was acutely and painfully aware of these data. Hospitals put quality improvement teams to work and hired consultants to gain a competitive advantage. Independent observers reported that quality results improved over time in Cleveland, and at a rate that exceeded positive trends noted throughout the country. Table 2 shows the percentage performance improvement for various diseases studied in Cleveland between 1993 and 1997 and clearly illustrates the rapid rate of improvement.

Over time, some participating hospitals began to accuse others of "gaming the system" and some publicly criticized the risk adjustment methodologies as "flawed". For example, they said that early discharge of terminally ill patients to hospice care was a "gaming" strategy resulting in shorter length of stay and lower death rates attributed to hospitals.

GCHQC data were perhaps as good as possible for the time, but not perfect. Harvard's Lisa Iezzoni reported that the Cleveland program used some of the most sophisticated clinical risk adjustment methods available. Would performance have improved as much without GCHQC? We may never know.

\section{WHAT HAPPENED?}

After the initial anxiety associated with public reporting, things quietened down and reporting became somewhat routine. However, during this time more than 30 independent hospitals went through mergers and consolidations or closure. As a result, the more than 30 independent hospitals in Cleveland at the beginning of the 1990s are now reduced to two large powerful delivery systems-one centred at the Cleveland Clinic and one at University Hospital-and, nationally, healthcare inflation returned to single digit annual increases. During this turn of events business turned its attention elsewhere, away from healthcare quality and costs. Health care and healthcare costs were no longer on the radar screen of corporate executives. Predictably, a political vacuum was created in Cleveland that shifted the balance of power away from businesses and back towards providers.

In December 1998 officials of the Cleveland Clinic informed GCHQC officials that their hospitals would no longer share patient information with the coalition. Effectively, the Cleveland Clinic and its nine hospitals representing one third of all participating Cleveland hospitals was withdrawing from GCHQC, thus bringing this noble and pioneering experiment to an abrupt end. ${ }^{1}$
Why did the Cleveland Clinic withdraw and, in so doing, contribute directly to the demise of an internationally recognized initiative? Clinic spokespersons said the program was too expensive; that it cost their nine hospitals $\$ 2$ million per year to participate. They wished to focus those funds on other internal quality initiatives. Other observers who may only be cynical and jealous of the Clinic's prestige said that GCHQC comparative data showed the Clinic to give good care but not noticeably better than several other hospitals in the area, and particularly not in the non-cardiac areas. These observers note that, at the same time, a prestigious poll of national rankings of hospitals by physician opinion published in World and News Report reported that the Cleveland Clinic was one of the very best hospitals in the country. These observers suggest that the Cleveland Clinic found these high opinions more to their liking than GCHQC's more scientific study.

\section{LESSONS LEARNED}

So, two decades later, what is the same and what is different in the Cleveland healthcare market? What's the same is easy. Annual double digit healthcare inflation has returned, the promise of managed care to control costs and monitor quality has failed, and health care is once again prominently displayed on the radar screen of business executives.

What's different? One concept (divide and conquer) is no longer a tenable employer strategy. Instead of more than 30 provider options there are now only two, albeit powerful providers (Cleveland Clinic and University Hospital). Instead of a highly sophisticated and comprehensive analysis and report of hospital performance being available there is a less sophisticated automaker's report in which the majority of hospitals do not participate. Lastly, 20 years ago Cleveland employers were in the power position; today, two powerful delivery systems will influence the market in Cleveland now and in the foreseeable future. And while other communities are moving forward in their attempts to bring sanity to healthcare delivery, what strategy is in the offing for Cleveland?

The legacies of GCHQC are several: its pioneering nature encouraged others to ask the questions and seek the answers about quality. The rich and robust database continues to support valuable scientific research that impacts on international health care. It showed that conventional adversaries (purchasers and providers) can work together, at least for a time, to improve health care in a community. Finally, stakeholders in Cleveland did benefit from this initiative by enjoying lower costs and improved quality of care and healthcare services.

The lessons to learn are that the consumer (patient, employee, employer, payer, health plan) must not blink; must remain steadfast in its demands for accountability; and must not allow a power vacuum to evolve between purchaser and provider.

\section{Authors' affiliations \\ D Neuhauser, Epidemiology and Biostatistics, Medical School, Case \\ Western Reserve University, Cleveland, USA \\ D L Harper, Florida Institute of Technology, Melbourne, Florida, USA}

Correspondence to: D Neuhauser, Epidemiology and Biostatistics, Medical School, Case Western Reserve University, Cleveland, $\mathrm{OH}$ 44106-4945, USA; dvn@cwru.edu

\section{REFERENCE}

1 Center for Studying Health System Change. Community Report: Cleveland, Ohio, Fall 2000. Washington, DC: The Center, 2000. 